\title{
Numerical Experiments Using a \\ Convective Flux Limiter on a Turbulent \\ Single-Mode Rayleigh-Taylor Instability
}

\author{
L.D. Cloutman
}

July 10, 2000

U.S. Department of Energy

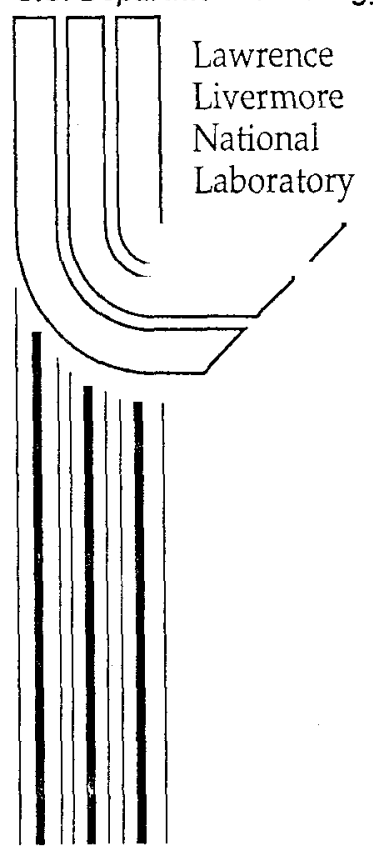




\section{DISCLAIMER}

This document was prepared as an account of work sponsored by an agency of the United States Government. Neither the United States Government nor the University of California nor any of their employees, makes any warranty, express or implied, or assumes any legal liability or responsibility for the accuracy, completeness, or usefulness of any information, apparatus, product, or process

disclosed, or represents that its use would not infringe privately owned rights. Reference herein to any specific commercial product, process, or service by trade name, trademark, manufacturer, or otherwise, does not necessarily constitute or imply its endorsement, recommendation, or favoring by the United States Government or the University of California. The views and opinions of authors expressed herein do not necessarily state or reflect those of the United States Government or the University of California, and shall not be used for advertising or product endorsement purposes. 
Lawrence Livermore National Laboratory

UCRL-ID-139735

July 2000

\title{
NUMERICAL EXPERIMENTS USING A CONVECTIVE FLUX LIMITER ON A TURBULENT SINGLE-MODE RAYLEIGH-TAYLOR INSTABILITY
}

\author{
Lawrence D. Cloutman \\ Lawrence Livermore National Laboratory \\ Livermore, California 94550
}

\begin{abstract}
Direct numerical simulation and large eddy simulations are powerful tools for studying turbulent flows. Unfortunately, they are computationally demanding in terms of run times, storage, and accuracy of the numerical method used. In particular, high order methods promise high accuracy on a given grid, but they often fail to deliver the expected accuracy due to dispersive truncation errors that appear as unphysical oscillations in the numerical solutions. This report describes a nonlinear flux limiter that has been applied to the second-order tensor viscosity method and markedly reduces the dispersive truncation errors. A Rayleigh-Taylor instability is simulated to show how well the flux limiter works.
\end{abstract}




\section{Introduction}

Direct numerical simulation (DNS) is a powerful tool for studying turbulent flows. By definition, a DNS resolves all flow features down to the microscale, so it is computationally challenging. It requires the largest and fastest computers, and even then such simulations are typically run with marginal resolution. A more commonly used tool is the large eddy simulation (LES), in which the large-scale flow features are resolved, and the effects of the subgrid-scale flow features are included by an eddy viscosity or other simple model. Both approaches make severe demands on the numerical algorithm if accuracy is to be attained.

There is an intermediate approach that has sometimes been used. A simulation is performed without a turbulence model, but without sufficient resolution to resolve the microscale. This is sometimes called a DNS in the literature, but I will make a distinction in this report and label this approach a basic simplified DNS (BSDNS). Apologists for this approach argue that one can avoid the expense and bother of using a turbulence model by relying on the numerical method used in the computational fluid dynamics (CFD) program to introduce the two major effects of a real turbulence model in cases where the resolution is inadequate to resolve all scales of motion. First, the grid imposes a high-wavenumber cutoff on the turbulence energy spectrum. Sccond, numerical diffusion provides a source of dissipation for the kinetic energy that cascades to the highest resolved wavenumbers. Indeed, it is easy to show that the numerical diffusion of first-order donor cell (upwind) differencing is the same order of magnitude as the Smagorinsky eddy viscosity, which has led to the suggestion of using donor cell (first order upwind) differencing as a "poor man's LES model." Unfortunately, as we shall see, the use of the BSDNS approach can lead to erroneous results. This is especially true in simulations based on the Eulcr equations, which allow "turbulence" to cascade all the way down to the grid scale regardless of how much resolution is used.

Regardless of which of the above approaches is used, the treatment of the convection terms is especially important. It is desirable to use methods that are at least second order in both space and time. It is important also that the methods be "monotonic," which means that positive definite quantities (such as density and temperature) remain nonnegative 
and that the method does not allow the unchecked growth of numerical oscillations due to dispersive truncation errors. These rcquirements are to some extent mutually exclusive since there is a tendency for high order methods to have significant dispersive errors.

In the end, what we really want are accurate solutions in the sense that the truncation errors are sufficiently small in magnitude. The fundamental requirement is that our numerical method be both consistent and stable so it can be convergent [1]. If the method we choose provides sufficient accuracy, the formal order of the method used is more a matter of politics and aesthetics than a technical issue. However, we know from experience that first order methods are usually inadequate. Consider the following example. The simplest monotonic method is donor cell. ${ }^{1}$ However, a careful accounting of the scaling of the eddy viscosity in the LUVD11 turbulence model $[2,3]$ and the Smagorinsky model $[4,5]$ shows it scales as $\delta x^{4 / 3}$ if the grid size $\delta x$ is in the inertial subrange of turbulence with a Kolmogorov spectrum. Donor cell has a strong diffusional truncation error with a diffusivity that scales as $\delta x$, which will get small more slowly than the LES terms as the grid is refined. Since the donor cell diffusivity is comparable to the LES viscosity even on fairly coarse grids, higher order methods with less numerical diffusion should be used.

There are many higher order methods with small numerical diffusion, but typically they have the unfortunate property that dispersive truncation errors causc significant numerical oscillations in the neighborhood of steep gradients. The oscillations tend to be worse the higher the order. What this means in practice is that higher order methods do not always guarantee high accuracy in the solution. In particular, they do not "increase resolution," as sometimes claimed in otherwise scholarly articles. There must be at least two zones across a flow feature regardless of the order of the method. Sccond, there is far more to the issue of accuracy than just the powers $\mathrm{x}$ of the grid size $\delta x$ and of the time step $\delta t$ that appoar as the truncation crror terms of the modificd equations. ${ }^{2}$ In poorly resolved flows, the high order derivatives can be huge, resulting in significant numerical errors in spite of the high formal order of accuracy.

\footnotetext{
${ }^{1}$ Donor cell differencing is monotonic only if the time step is sufficiently small. The time step limit for monotonicity is less than that for formal linear stability, as we shall see in the next section.

${ }^{2}$ Modified equations are the differential equations obtained by Taylor series expansion of the difference equations. For a consistent method, the zeroth order terms are just the original differential equations.
} 
A varicty of approaches for alleviating this accuracy problem have been suggested, and it is beyond the scope of this report to discuss them. What we need is a simple but effective flux limiter that can be easily retrofitted to existing CFD codes. In the next section, we describe such a method, which we call the convection-limiting accuracy maintenance (CLAM) method. In the third section, we apply the CLAM method to a classical Rayleigh-Taylor instability. Conclusions are presented in the fourth section.

\section{The CLAM Method}

There are many ways to reduce dispersively driven numerical oscillations, some of them quite sophisticated, complex, and difficult to understand. What I want is something simple and economical to use, casy to understand and straightforward to implement. An attractive approach that meets these requirements is to use convective fluxes computed as a lincar combination of high order fluxes and donor cell fluxes determined by a local prescription of how much of each to use such that nemerical oscillations are controlled. Among the earliest attempts was an empirically-based method described by Clark and Hill [6, 7] and modified by Smolarkiewicz [8]. Later came the FRAM method of Chapman [9]. He used a test on a Lagrangian estimate of the local density field to switch between a high order method and donor cell differencing in such a way that extrema were not unphysically enhanced. Later this method was modified to produce less overall numerical diffusion in arbitrary LagrangianEulcrian (ALE) codes [10]. However, while that method works quite well, it is not as readily implemented in pure Eulerian codes. Since there is a current need for reducing dispersive errors in the COYOTE Eulcrian code [11], I developed an improved version of the Clark-Hill approach, the CLAM method.

The CLAM method is based on a hybrid convective flux obtained by a linear combination of a flux from a high order method with the flux from donor cell differencing. We illustrate the method by considering the tensor viscosity (TV) method [12], which is formally second order in both space and time. It has the considerable advantages of being a single-step explicit method and accurate in cases where dispersive truncation errors are small. However, it has a tendency to introduce spurious oscillations in regions of steep gradients, which compromises the accuracy despite being second order. The truncation errors in high-order 
methods are not necessarily small. Going to even higher orders does not necessarily help. Experience has shown, for example, that the third order QUICK method [13] has an even more pronounced tendency to produce oscillations.

Let us begin describing the CLAM filter by considering the advection equation for a scalar variable $\phi$ :

$$
\frac{\partial \phi}{\partial t}+\frac{\partial \phi u}{\partial x}=S_{\phi}
$$

Let $\phi_{i}$ be the value of $\phi$ at cell center $i$, and let the convective fluxes $F_{i-1 / 2}$ and velocities $u_{i-1 / 2}$ be defined on cell edges $i-1 / 2$. All quantities are evaluated at time level $n$ unless denoted by the superscript $n+1$. Then we use the explicit finite difference approximation

$$
\begin{gathered}
\phi_{i}^{n+1}=\phi_{i}-\frac{\delta t}{\delta x_{i}}\left(F_{i+1 / 2}-F_{i-1 / 2}\right)+\delta t S_{\phi i} . \\
F_{i-1 / 2}=(\phi u)_{i-1 / 2}=(1-\alpha-\beta) F_{i-1 / 2}^{C}+\alpha F_{i-1 / 2}^{D C}+\beta F_{i-1 / 2}^{T V} \\
=0.5 u_{i-1 / 2}\left(\phi_{i-1}+\phi_{i}\right)-0.5\left(\alpha\left|u_{i-1 / 2}\right| \delta x_{i-1 / 2}+\beta u_{i-1 / 2}^{2} \delta t\right)\left(\frac{\phi_{i}-\phi_{i-1}}{\delta x_{i-1 / 2}}\right) .
\end{gathered}
$$

Here we have made use of the following definitions for the centered, donor cell, and TV fluxes:

$$
\begin{gathered}
F_{i-1 / 2}^{C}=0.5\left(\phi_{i}+\phi_{i-1}\right) u_{i-1 / 2} \\
F_{i-1 / 2}^{D C}=0.5\left[\phi_{i}\left(u_{i-1 / 2}-\left|u_{i-1 / 2}\right|\right)+\phi_{i-1}\left(u_{i-1 / 2}+\left|u_{i-1 / 2}\right|\right)\right] \\
=F_{i-1 / 2}^{C}-\frac{\delta x_{i-1 / 2}\left|u_{i-1 / 2}\right|}{2} \frac{\left(\phi_{i}-\phi_{i-1}\right)}{\delta x_{i-1 / 2}} \\
F_{i-1 / 2}^{T V}=0.5\left(\phi_{i}+\phi_{i-1}\right) u_{i-1 / 2}-\frac{\delta t u_{i-1 / 2}^{2}}{2} \frac{\left(\phi_{i}-\phi_{i-1}\right)}{\delta x_{i-1 / 2}} \\
=F_{i-1 / 2}^{C}-\frac{\delta t u_{i-1 / 2}^{2}}{2} \frac{\left(\phi_{i}-\phi_{i-1}\right)}{\delta x_{i-1 / 2}}
\end{gathered}
$$

In these equations, $0 \leq \alpha \leq 1$ is the donor cell fraction, $0 \leq \beta \leq 1$ is the tensor viscosity fraction, and $\xi=1-\alpha-\beta$ is the centered differencing fraction (normally set to zero). Normally, we run the code with $\alpha=0$ and $\beta=1$ whenever possible since this combination is second order accurate in space and time. However, it is prone to producing nonphysical oscillations in solutions with stcep gradients. The procedure then is to make $\alpha$ nonzcro but as small as possible while still suppressing the oscillations. The idea is to damp the 
oscillations while introducing only the minimum amount of first order diffusion from the donor cell component.

This hybrid flux expression has some desirable properties. First, all three component fluxes are consistent, so the total flux expression is consistent. It also has acceptable stability properties. If we use centered differencing $(\alpha=\beta=0)$, the difference cquations 2 and 3 are unconditionally unstable even though it is formally second order in space. This is why we almost always run with $\xi=0$. However, it is convenient to write the fluxes in terms of centered differencing plus a diffusion-like correction term, so we retain the option of using centered differences for testing and instructional purposes. The other two flux expressions are conditionally stablc, and therefore convergent (at least for linear problems in which $u$ is a constant) [1]. In both cases, the formal stability limit is $\chi \equiv|u| \delta t / \delta x_{i}<1$. In practice, we can seldom get close to this limit when $u$ is not constant, and we must use $\chi \approx 0.2$.

There are also some interesting monotonicity considerations. By monotonic, we mean a method that will not enhance extrema of the solution in a nonphysical way or introduce spurious extrema. In other words, the numerical method will not produce "wiggles" due to dispersive truncation errors. The TV method is well known to be nonmonotonic even for constant $u$, which can introduce substantial crrors even though the method is formally second order in space and time. Donor coll is monotonic under certain conditions, and these conditions are related to the fact that the linear stability limit cannot be approached for most problems. For constant $u$, donor cell is stable and monotonic up to the linear stability limit. However, it is easy to see that the situation is different for variable $u$. Consider the case $u_{i-1 / 2}=(-1)^{i} u_{0}$, where $u_{0}>0$ is a constant. Assume also that $\phi>0$ is a constant. The correct solution for this problem is $\phi$ going to zero in those cells for which the velocity on the two cell faces is outward. This insures the positive-definiteness of $\phi$. However, it is easy to see that if $\chi>0.5, \phi$ will become negative on the first cycle. While the scheme remains stable, it produces an unphysical, nonmonotonic result. The time step limit for monotonicity becomes even more restrictive in two and three dimensions. It is easy to see that if the outward flux times the cell face area has the same magnitude on every cell face, the monotonicity limit is $\chi<1 / N_{f}$ where $N_{f}$ is the total number of cell faces.

The basic idea behind the CLAM method is to make $\alpha$ and $\beta$ a function of space 
and time and to use simple tests to choose the locally required value of $\alpha$ needed to prevent numerical oscillations. The goal is to introduce strong smoothing only where and when it is actually needed, thereby minimizing the first order diffusive errors.

CLAM operates in a sequence of simple steps. We begin by specifying constant values of $\alpha$ and $\beta$ that will be used for the baseline, lowest-diffusion method to be applied everywhere that CLAM makes no changes. The present implementation is such that the donor cell fraction is never less than $\alpha$, and $\xi$ is always the same constant value regardless of what CLAM does. The next step is to compute a provisional value of the donor cell fraction on each cell face using an empirically derived expression. Define a new variable $\varphi$ such that $\phi=\varphi=\rho$ for the density and $\phi=\rho \varphi$ for all other variables. We choose

$$
\hat{\alpha}_{i-1 / 2}=A\left|\frac{\Delta \varphi}{\bar{\varphi}}\right|+B\left(\frac{\Delta \varphi}{\bar{\varphi}}\right)^{2}+C\left|\frac{\Delta^{2} \varphi}{\bar{\varphi}}\right|+D\left(\frac{\Delta^{2} \varphi}{\bar{\varphi}}\right)^{2}+E\left|\frac{\Delta^{3} \varphi}{\bar{\varphi}}\right|+F\left(\frac{\Delta^{3} \varphi}{\bar{\varphi}}\right)^{2},
$$

where $A, B, C, D, E$, and $F$ are constants and

$$
\begin{gathered}
\Delta \varphi=\varphi_{i}-\varphi_{i-1} \\
\Delta^{2} \varphi=\max \left(\left|\varphi_{i}-2 \varphi_{i-1}+\varphi i-2\right|,\left|\varphi_{i+1}-2 \varphi_{i}+\varphi i-1\right|\right) \\
\Delta^{3} \varphi=\varphi_{i+1}-3 \varphi_{i}+3 \varphi_{i-1}-\varphi_{i-2}
\end{gathered}
$$

We do this calculation for $\varphi=\rho$, the specific internal energy $I$, and the temperature $T$, and then take the largest of the three values of $\hat{\alpha}_{i-1 / 2}$ as the provisional donor cell fraction on that cell face.

There are several different options for $\bar{\varphi}$ :

$$
\begin{aligned}
\bar{\varphi} & =\text { constant } \\
& =\left|\varphi_{i}\right|+\left|\varphi_{i-1}\right|+\epsilon \\
& =\min _{\text {all } i}\left(\left|\varphi_{i}\right|\right) \\
& =\min _{i-1 \leq j \leq i}\left(\left|\varphi_{j}\right|\right) \\
& =|| \varphi_{i}|+| \varphi_{i-1}\left|-2 \varphi_{0}\right|+\epsilon .
\end{aligned}
$$

The examples in the next section use the second line. The use of a constant value, perhaps approximately equal to the minimum value of $\varphi$ in the mesh, is probably better in problems 
where $\varphi$ varies by a large factor, such as in the presence of a strong shock wave. The last line is probably not a good choice for many problems since it has the potential of becoming zero for an inauspicious choice of the constant $\varphi_{0}$.

The motivation for this form is heuristic in nature. Smolarkiewicz [8] suggests $B=1.0$ and zero for all the other constants. In our experience, this choice to provides too little smoothing, at least for the Rayleigh-Taylor instabilities to be described in the next section. The terms proportional to the first or second power of the slope are included because the numerical oscillations occur where steep gradionts are convected in the direction of the gradient vector (that is, normal to an interface). However, oscillations can extend a number of zones away from the steep part of the gradient. The second differences are included because these are effective at picking up regions where the solution has an every-other-eell oscillation, and we want to use the donor cell numerical diffusion to damp these. Dispersive truncation errors are responsible for the oscillations with a period of 5 to 7 cells, and these are proportional to third derivatives. Hence, the inclusion of the $\Delta^{3}$ terms.

Once we have a provisional $\alpha$, we apply some cutoffs to it to make sure it is within reasonable limits. Introduce two cutoff values, $\alpha_{0}$ and $\alpha_{1}$ such that $\xi \leq \alpha_{1} \leq \alpha \leq \alpha_{0}$. Then if $\hat{\alpha}_{i-1 / 2}>\alpha_{0}$, then $\alpha_{i-1 / 2}=1-\xi$. If $\hat{\alpha}_{i-1 / 2}<\alpha_{1}$, then $\alpha_{i-1 / 2}=\alpha$. Otherwise, $\alpha_{i-1 / 2}=\hat{\alpha}_{i-1 / 2}$. Note that if $\alpha_{0}=\alpha_{1}$, the method switches back and forth between pure donor cell and pure TV, similar to the FRAM method.

Once all this has been done, we can do one final thing. For zones in which any of the positive-definite cell-centered quantities $\rho, I, T$, or any of the species densities are negative, set the $\alpha_{i-1 / 2}=1.0$ on all faces of that cell.

\section{Rayleigh-Taylor Instability}

In this report, we consider a classical Rayleigh-Taylor instability simulated with the COYOTE computer program [11]. COYOTE solves the compressible multicomponent NavierStokes equations for both cold and reactive flows in two-dimensional Cartesian and axisymmetric cylindrical geometries. It includes the LUVD11 turbulence model $[2,3]$ and the Smagorinsky model $[4,5]$ as options. The former subgrid-scale (SGS) turbulence model employs a single transport equation for the turbulence kinetic energy density. The latter model 
cmploys an algebraic SGS eddy viscosity.

In the classical Rayleigh-Taylor instability, a layer of dense fluid is placed on top of a layer of less dense fluid. Buoyancy forces cause an initial perturbation of the interface to grow, allowing the denser fluid to fall downwards, displacing the lightor fluid upwards at an increasing ratc. Eventually, for immiscible fluids, the system ends up at rest with the denser fluid on the bottom, and the lighter fluid on the top. For miscible fluids such as the gases considered here, some mixing at the molecular level will occur. In this example, the flow becomes turbulent, which enhances the molecular mixing. The final state will be a stratified mixture whose composition profile will depend on the details of the flow.

The initial condition considered in this report has an interface perturbed by a single Fouricr mode as shown in Figure 1. Gas with a density of $1.57 \times 10^{-2} \mathrm{~g} / \mathrm{cm}^{3}$ lics above a layer of gas with a density of $1.0 \times 10^{-2} \mathrm{~g} / \mathrm{cm}^{3}$, which gives an Atwood number of At $=0.222$. The temperature is a uniform $287.7 \mathrm{~K}$. Both fluids are perfect gases with $\gamma=5 / 3$ and with molecular weights of 15.7 and 10.0 for the heavy and light gases respectively. A gravitational acceleration of $6.86 \times 10^{4} \mathrm{~cm} / \mathrm{s}^{2}(70 \mathrm{G})$ points downward. Rigid frec-slip boundaries are used on all four walls. Transport cocfficients are computed from the Lennard-Jones model [14] using some arbitrary but typical values for the potential parameters: $\sigma=2.576$ and $3.621 \AA$ and $\epsilon / k=10.20$ and 97.53 for spccies 1 (heavy) and 2 (light), respectively.

The dimensionless parameters for this problem are estimated from the solution at $40 \mathrm{~ms}$ when the instability is well developed but has not becn disrupted by the heavy fluid hitting the bottom of the grid. Adiabatic sound speeds are $5.0 \times 10^{4} \mathrm{~cm} / \mathrm{s}$ and $6.3 \times 10^{4} \mathrm{~cm} / \mathrm{s}$ in the heavy and light gases respectively. The highest speeds obtained at any time are on the order of $400 \mathrm{~cm} / \mathrm{s}$, so the maximum Mach number is on the order of 0.01 . There is no unique Reynolds number associated with this problem as the velocity changes dramatically with time. However, using a speed of $300 \mathrm{~cm} / \mathrm{s}$, the viscosity of the less viscous gas $\left(0.01 \mathrm{~cm}^{2} / \mathrm{s}\right)$, and the $7.3 \mathrm{~cm}$ initial perturbation wavelength for the length scale, the Reynolds number is $2.2 \times 10^{4}$. If we choose the higher viscosity $\left(0.024 \mathrm{~cm}^{2} / \mathrm{s}\right)$, the Reynolds number at $40 \mathrm{~ms}$ is $10^{4}$, which still indicates that a turbulent flow should develop from the initially laminar instability. It would take a grid with one or two thousand zones on each side to perform a DNS. 
We implemented the CLAM algorithm in COYOTE and produced five planar twodimensional solutions with different convection algorithms and turbulence models. Three solutions were BSDNSs without a turbulence model. These used donor cell differencing, TV, and TV with the CLAM method (CLAM-TV). The other two solutions were done using the LUVD11 one-equation turbulence transport model $[2,3]$. One of those used TV, and the other used CLAM-TV. In all cases, the 7.3 by $8.8 \mathrm{~cm}$ mesh was covered by a uniform grid of $73 \times 88$ zones. The initial and boundary conditions were the same as in a previous study [15].

\subsection{Donor Cell Solution}

Figures 2 through 4 show the total density, temperature, and mass fraction contour plots for the donor cell solution. The bubbles and spikes are almost symmetric about the midplane of the mesh. The initial interface, which was onc zone wide, has been broadened significantly by numerical diffusion, but it still exhibits the expected vortex pair shed by the tip of the spike. Donor cell differencing has a numerical diffusivity of approximately

$$
\nu_{i, \mathrm{dc}}=0.5\left|u_{i}\right| \delta x_{i}\left(1-\frac{\left|u_{i}\right| \delta t}{\delta x_{i}}\right)
$$

in the $x_{\imath}$ direction [8]. Since the itcrative algorithm that makes COYOTE partially implicit begins to become inefficient when acoustic waves can travel more than three to five zones in one time step, this problem was run with the factor in parentheses greater than about 0.97 . Therefore, we shall neglect it, and we estimate the numerical viscosity on the finest grid to be $4.4 \mathrm{~cm}^{2} / \mathrm{s}$ in the fastest part of the flow. This is over two orders of magnitude larger than the physical viscosity, and it is sufficient to make the advection algorithm monotonic. The effective Reynolds number of the simulation is on the order of 100 , and we have a solution that appears to be laminar.

The donor cell method is monotonic for the small time steps used in this calculation.

This can be seen in the density plot, where the max and min values are $1.57 \times 10^{-2}$ and $1.0 \times 10^{-2} \mathrm{~g} / \mathrm{cm}^{3}$, which are the initial values of the two fluid densities. There are no overshoots or undershoots. The temperature fluctuations divided by the initial temperature are on the order of $10^{-4}$, which is the square of the Mach number, just as expected. Although the problem is isothermal to a high degree of approximation, the internal energies in the two 
fluids are $2.29 \times 10^{9}$ and $3.59 \times 10^{9} \mathrm{crgs} / \mathrm{g}$, and the contours look the same as the density contours. The difference is due to the differing molecular weights. The maximum velocity is $293 \mathrm{~cm} / \mathrm{s}$.

\subsection{Tensor Viscosity Solution}

Figures 5 through 7 were calculated by the TV method with neither CLAM method nor turbulence model. The numerical diffusivity is tensorial in nature, ${ }^{3}$

$$
\overrightarrow{\vec{\nu}}_{\mathrm{tv}}=0.5 \delta t \mathbf{u u}
$$

which is a factor of $\chi$ smaller than the donor cell viscosity. Much of this positive diffusivity is used to cancel diffusive truncation crrors with negative diffusivities. Comparison of the mass fraction plots figures 4 and 7 shows the greatly reduced diffusion in the TV solution. The width of the interface is much smaller and the production of vorticity is much larger in the latter plot.

As discussed in a previous report, this solution has very poor accuracy [15]. Not only is the shape of the interface all wrong, the dispersive errors are enormous. Compared to the initial values, the maximum density has gone from $1.57 \times 10^{-2} \mathrm{~g} / \mathrm{cm}^{3}$ to $4.08 \times 10^{-2}$, and the minimum from $1.0 \times 10^{-2}$ to $4.35 \times 10^{-3}$. The isothermal $287.7 \mathrm{~K}$ fluid now has temperatures from 110.9 to $1078.1 \mathrm{~K}$. The mass fraction shows only a small amount of under- and overshooting. Also, the peak speed is $543 \mathrm{~cm} / \mathrm{s}$, which is almost a factor of two faster than in the donor coll solution. The accuracy can be improved by increased resolution, but even zones 0.25 as wide still have unacceptable errors and show a lack of grid independence. [15]. Notice also that the time step has dropped from $25 \mu \mathrm{s}$ down to a little over $4 \mu$ s due to the need to resolve the fine-scale numerical artifacts. It would be pointless folly to perform a statistical analysis of the "turbulence" in this calculation.

\subsection{CLAM Method Solution}

Figures 8 through 10 were calculated with CLAM-TV and no turbulence model. The model constants are $A=5.0, B=D=F=1.0, C=E=3.0, \alpha_{0}=0.8$, and $\alpha_{1}=0.011$. The

\footnotetext{
${ }^{3}$ The donor cell diffusivity may also be written in matrix form, with diagonal elements given by equation 12 and with zeroes off the diagonal [12].
} 
overall shape of the interface is much more like that of the donor cell solution, which is quite reasonable. But now the interface thickness is noticably less, and the vortex at the edge of the mushroon cap is much stronger. These are due to the reduced numerical diffusion of the CLAM method compared with full donor coll differencing.

Although there are still residual numerical oscillations, the amplitude is greatly reduced to just a few percent. The densities now lie between $9.86 \times 10^{-3}$ and $1.66 \times 10^{-2}$ $\mathrm{g} / \mathrm{cm}^{3}$, and the temperatures lic between 272.3 and $295.9 \mathrm{~K}$. The small oscillations in the mass fraction have been reduced to an even more insignificant level. The maximum fluid speed is $348 \mathrm{~cm} / \mathrm{s}$.

\subsection{LES Model Solution}

Figures 11 through 13 show the solution for the TV method plus the LUVD11 turbulence model. LUVD11 is constructed so that for turbulence that has attained equilibrium between production and decay, one obtains the same eddy viscosity as the Smagorinsky model. The turbulence length scale is assumed to be $1.94 \delta x$. However, the LUVD11 model produces too much smoothing in this calculation. The shape of the spike is odd for reasons that are not completely understood. Perhaps it is the strong cross-stream diffusion along the sides of the spike. In any case, we have seen that resolution improves this situation [15].

The interface thickness is as large as for donor cell differencing, and the vortex formation is even more suppressed by the cross-stream diffusion. The peak velocity is $296 \mathrm{~cm} / \mathrm{s}$. Temperature fluctuations are confined to the tips of the bubbles and spike. The fluctuation has been reduced to $1 \mathrm{~K}$ in the bubble and $+1.2,-4.5$ in the spike, giving a range of 283.2 to $289.0 \mathrm{~K}$ over the whole mesh. The mass fraction is strictly monotonic.

\subsection{CLAM-TV Solution with LES}

Figures 14 through 16 show the plots for the solution performed with CLAM-TV and LUVD11. The shape of the interface is more nearly normal than in the previous case shown in figures 11 through 13 . This is most likely due to the reduction in the turbulence kinetic encrgy production caused by suppression of the artificial small-scale fluctuations by CLAM. This is consistent with the observation that the interface is not as thick, and the vortices in 
the mushroom caps are stronger. Indeed, plots of the turbulence kinetic energy show similar distributions, but this case has a peak value that is 10 percent smaller.

The peak velocity is $293 \mathrm{~cm} / \mathrm{s}$. The scalar ficlds are no longer quite monotonic, a result of the slight reduction in the eddy viscosity. Density overshoots are $+5.2 \times 10^{-4}$ (3.3 percent) to $-6.1 \times 10^{-5}$ (0.4 percent). Temperatures range from 278.6 to 291.1 , for fluctuations of -9.1 to $+3.4 \mathrm{~K}$.

It is interesting that with the cxception of unmodificd TV, these calculations all predict pretty much the same amplitude of the instability regardless of the fine details. This insensitivity of the thickness of the mixed layer to details of numerical method and turbulence model suggests that the growth rate of Rayleigh-Taylor instabilities may not be a good diagnostic for testing turbulence models.

\section{A Curious Result}

Figure 17 shows the horizontally averaged mass fraction of the heavy species as a function of height. Flux-limitcd runs with and without the turbulence model both produce a pronounced slope reversal in the middle of the mixed zone. This feature can easily be understood by looking at the mass fraction plots, which show heavy fluid accumulating at the spike tip and light fluid piling up at the bubble tips. For a single-mode instability at this stage of its development, this is a reasonable result. We point out, however, that this slope reversal cannot be predicted by a one-dimensional model, such as the $k-\epsilon$ model, which uses a diffusion approximation for the mass fluxes. This is strictly a convective effect. Preliminary singly and multimode simulation suggests that the reversal may not be present at late times, or even never present if the flow is more "turbulent" and less organized as in the case of a multimode initial condition. This point is being studied further.

\section{Conclusions}

The primary objective of this study is to illustrate the effects of numerical methodology and turbulence models on a gencric Rayleigh-Taylor instability. This was accomplished with the COYOTE CFD program using a variety of different numerical methods and turbulence 
models to produce five numerical solutions. On the basis of these simulations, wo have arrived at the following conclusions:

- The TV method by itself produced unacceptable results due to the dispersive truncation errors, which are a side effect of the low numerical diffusion. We successfully introduced a very simple, heuristic flux limiter, the CLAM method, that produced a much more realistic simulation of a Rayliegh-Taylor instability. Even though the CLAM method as presented here did not completely eliminate the dispersive oscillations, it markedly reduced them.

- The CLAM method is easy to implement in any code in which the convective fluxes are computed as a mixture of a high order method and donor cell. This is especially advantageous in existing codes where other types of flux limiters may require extensive code modifications. Also, CLAM is computationally inexpensive, mathematically consistent and stable, and can produce no more diffusion than donor cell differencing.

- The concept of the BSDNS (an unresolved DNS) was tested, and the poor performance of the unmodified TV method strongly suggests that if a BSDNS is to be reliable, the convective terms of the CFD algorithm must be monotonic, either by the construction of the difference method or by the use of flux limiters.

- Another approach, which we have not tried, is to use the unmodified TV method, but artificially increase the value of the molecular viscosity to the point that it provides a reasonable amount of dissipation at the grid scale.

- The insensitivity of the thickness of the mixed layer to details of numerical method and turbulence model suggests that the growth rate of Rayleigh-Taylor instabilities is not a good diagnostic for testing turbulence models.

\section{Acknowledgements}

It is a pleasure to acknowledge several useful conversations with John Ramshaw. This work was performed under the auspices of the U. S. Department of Energy and University of California Lawrence Livermore National Laboratory under contract No. W-7405-Eng-48. 


\section{References}

[1] R. D. Richtmyer, Difference Methods for Initial-Value Problems, Interscience Publishers, NY, 1957.

[2] Cloutman, L. D., "The LUVD11 Large Eddy Simulation Model," Lawrence Livermore National Laboratory report UCRL-ID-107128, 1991.

[3] L. D. Cloutman, "Numerical simulation of the heat transfer and decay of turbulent swirling flow," Comput. \& Fluids, 17, 437 (1989).

[4] J. Smagorinsky, "General circulation experiments with the primitive equations I. The basic expcriment," Mon. Weather Rev. 91, 99 (1963).

[5] J. W. Deardorff, "On the magnitude of the subgrid scale eddy coefficient," J. Comput. Phys. 7, 120 (1971).

[6] T. L. Clark and W. D. Hall, "A numerical cxperiment on stochastic condensation theory," J. Atmos. Sci. 36, 470 (1979).

[7] T. L. Clark, "Numerical simulations with a three-dimensional cloud model: Lateral boundary condition experiments and multicellular severe storm simulations," J. Atmos. Sci. 36, 2191 (1979).

[8] P. K. Smolarkiewicz, "A simple positive definite advection scheme with small implicit diffusion," Mon. Weather Rev. 111, 479 (1983).

[9] M. Chapman, "FRAM - Nonlinear damping algorithms for the continuity equation," J. Comput. Phys. 44, 84 (1981).

[10] L. D. Cloutman, "A convective flux limiter for non-Lagrangian computational fluid dynamics," J. Comput. Phys. 73, 349 (1987).

[11] Cloutman, L. D., "COYOTE: A Computer Program for 2-D Reactive Flow Simulations," Lawrence Livermore National Laboratory report UCRL-ID-103611, 1990. 
[12] J. K. Dukowicz and J. D. Ramshaw, "Tensor viscosity method for convection in numerical fluid dynamics," J. Comput. Phys. 32, 71 (1979).

[13] B. P. Leonard, "Simple high-accuracy resolution program for convective modelling of discontinuities," Int. J. Numer. Methods Fluids 8, 1291 (1988).

[14] L. D. Cloutman, "A Database of Selected Transport Coefficients for Combustion Studies," Lawrence Livermore National Laboratory report UCRL-ID-115050, 1993.

[15] L. D. Cloutman, "Numerical Expcriments With a Turbulent Single-Mode RayleighTaylor Instability," Lawrence Livermore National Laboratory report UCRL-ID-138595, 2000. 


\section{$Y 1$ cycle $=0 \mathrm{t}=0.000000 \mathrm{D}+00 \mathrm{dt}=1.000000 \mathrm{D}-06$ $\max =1.000000 \mathrm{D}+00 \mathrm{~min}=0.000000 \mathrm{D}+00 \mathrm{dq}=1.000000 \mathrm{D}-01$}

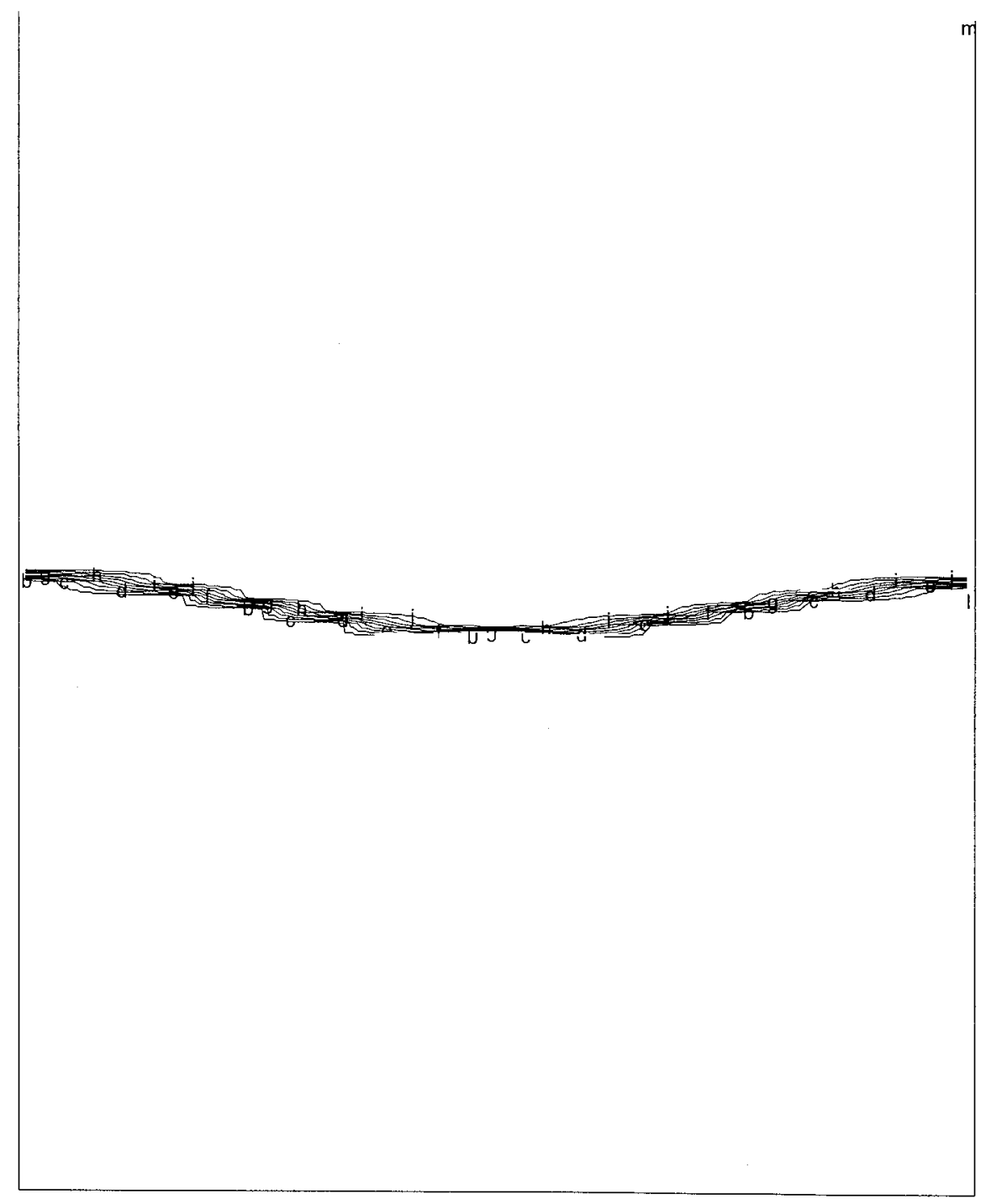

Figure 1: Mass fraction contours for species 1 at $0 \mathrm{~ms}$. The heavy gas (species 1 ) is on the top. The grid is $7.3 \mathrm{~cm}$ wide by $8.8 \mathrm{~cm}$ tall, and it is covered with $73 \times 88$ uniform zones. The initial interface is $y=4.4+0.2 \cos (2 \pi x / 7.3)$. In all plots, the contour labels $b$ through $j$ denote contour values of 0.1 through 0.9 in steps of 0.1 . The legend at the top of the figure gives the cycle number, the problem time, the current time step, the maximum function value (max), the minimum function value (min), and the contour spacing. All units are cgs. 
Density cycle $=2133 \mathrm{t}=4.001435 \mathrm{D}-02 \mathrm{dt}=2.500000 \mathrm{D}-05$ $\max =1.570044 \mathrm{D}-02 \mathrm{~min}=9.999801 \mathrm{D}-03 \mathrm{dq}=5.700644 \mathrm{D}-04$

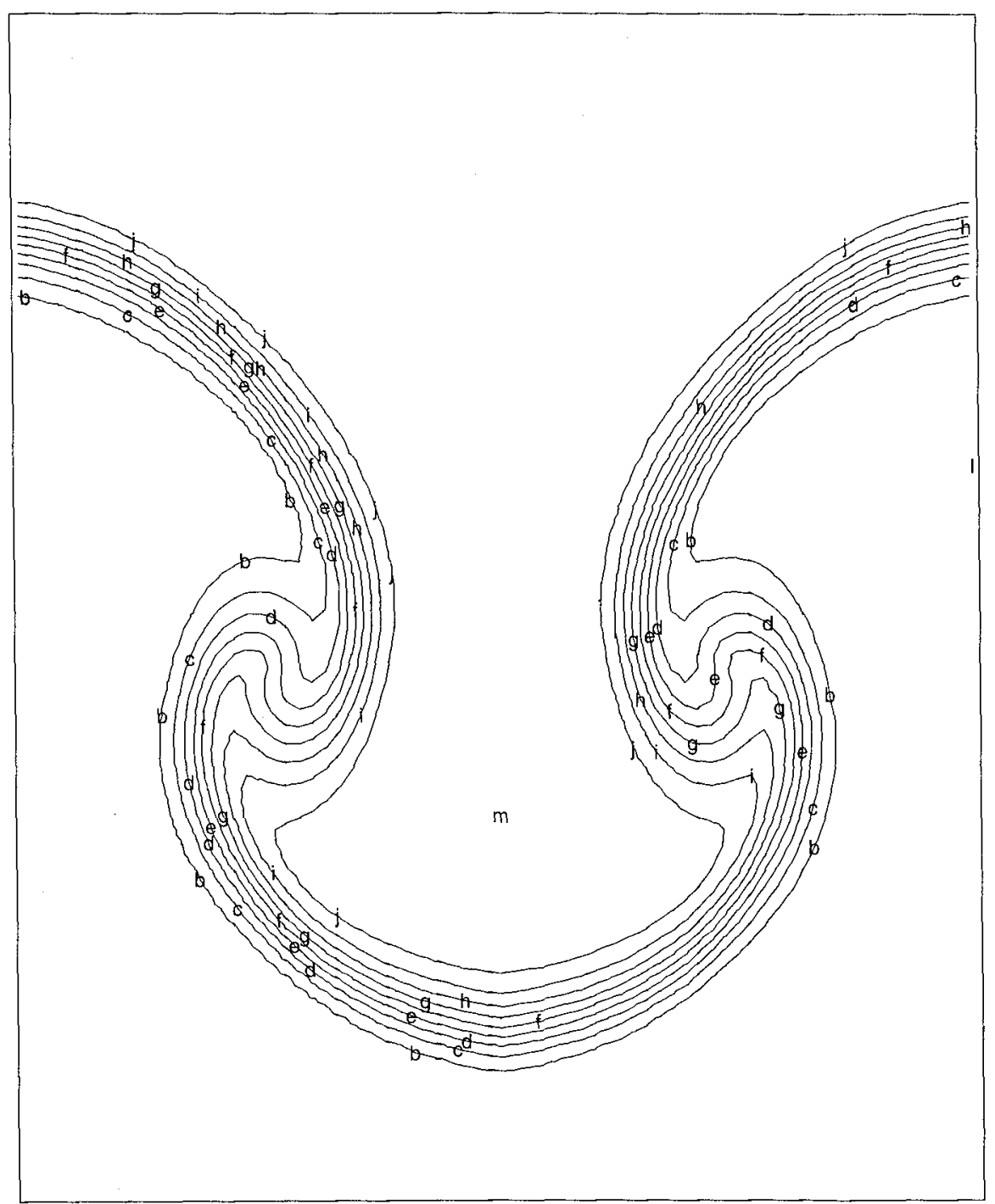

Figure 2: Total density contours at $40 \mathrm{~ms}$ using donor cell differencing. 
Temper cycle $=2133 \mathrm{t}=4.001435 \mathrm{D}-02 \mathrm{dt}=2.500000 \mathrm{D}-05$ $\max =2.877186 \mathrm{D}+02 \mathrm{~min}=2.876815 \mathrm{D}+02 \mathrm{dq}=3.714991 \mathrm{D}-03$

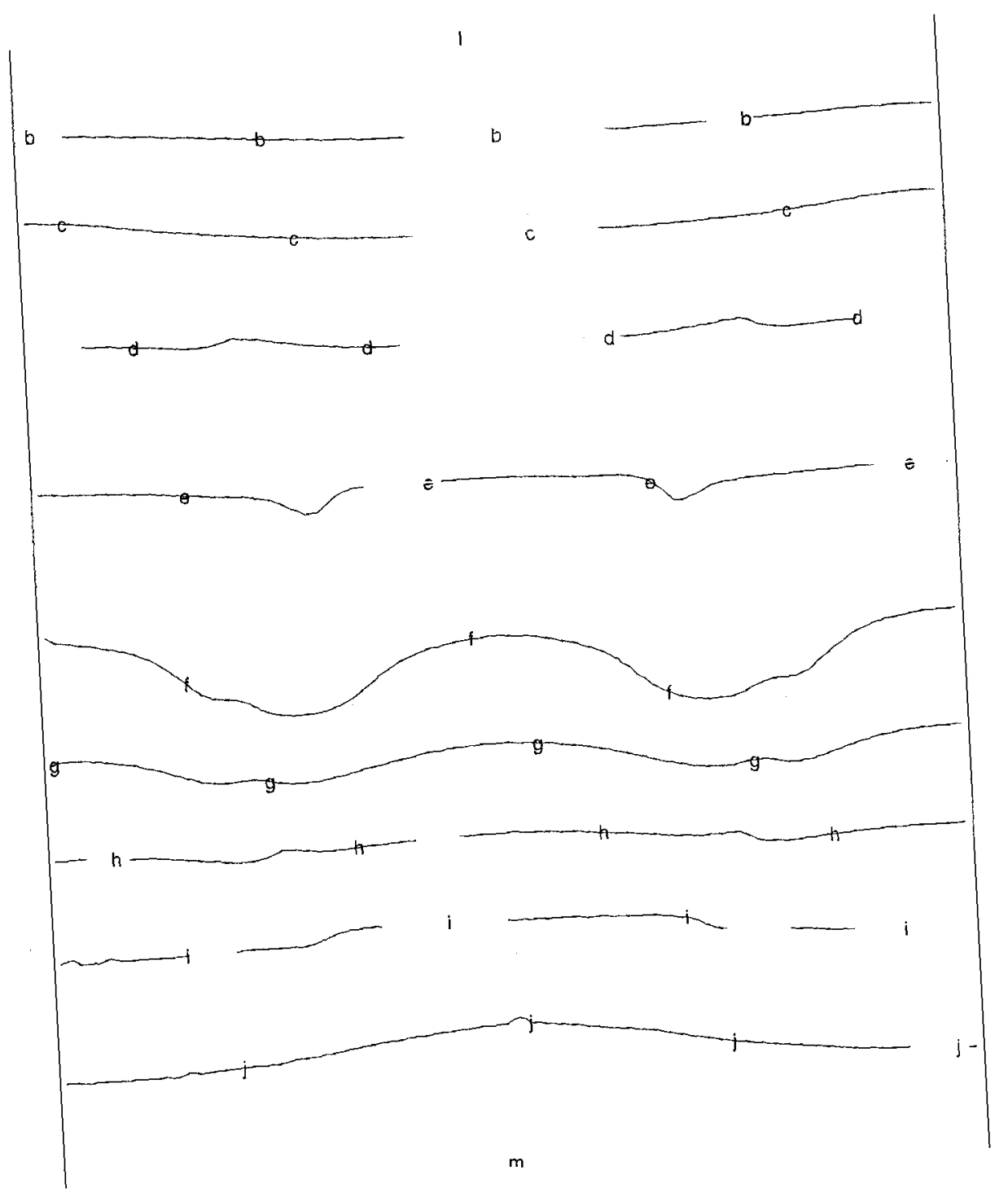

Figure 3: Temperature contours at $40 \mathrm{~ms}$ using donor cell differencing. 
$\mathrm{Y} 1$ cycle $=2133 \mathrm{t}=4.001435 \mathrm{D}-02 \mathrm{dt}=2.500000 \mathrm{D}-05$
$\max =1.000000 \mathrm{D}+00 \mathrm{~min}=2.358746 \mathrm{D}-41 \mathrm{dq}=1.000000 \mathrm{D}-01$

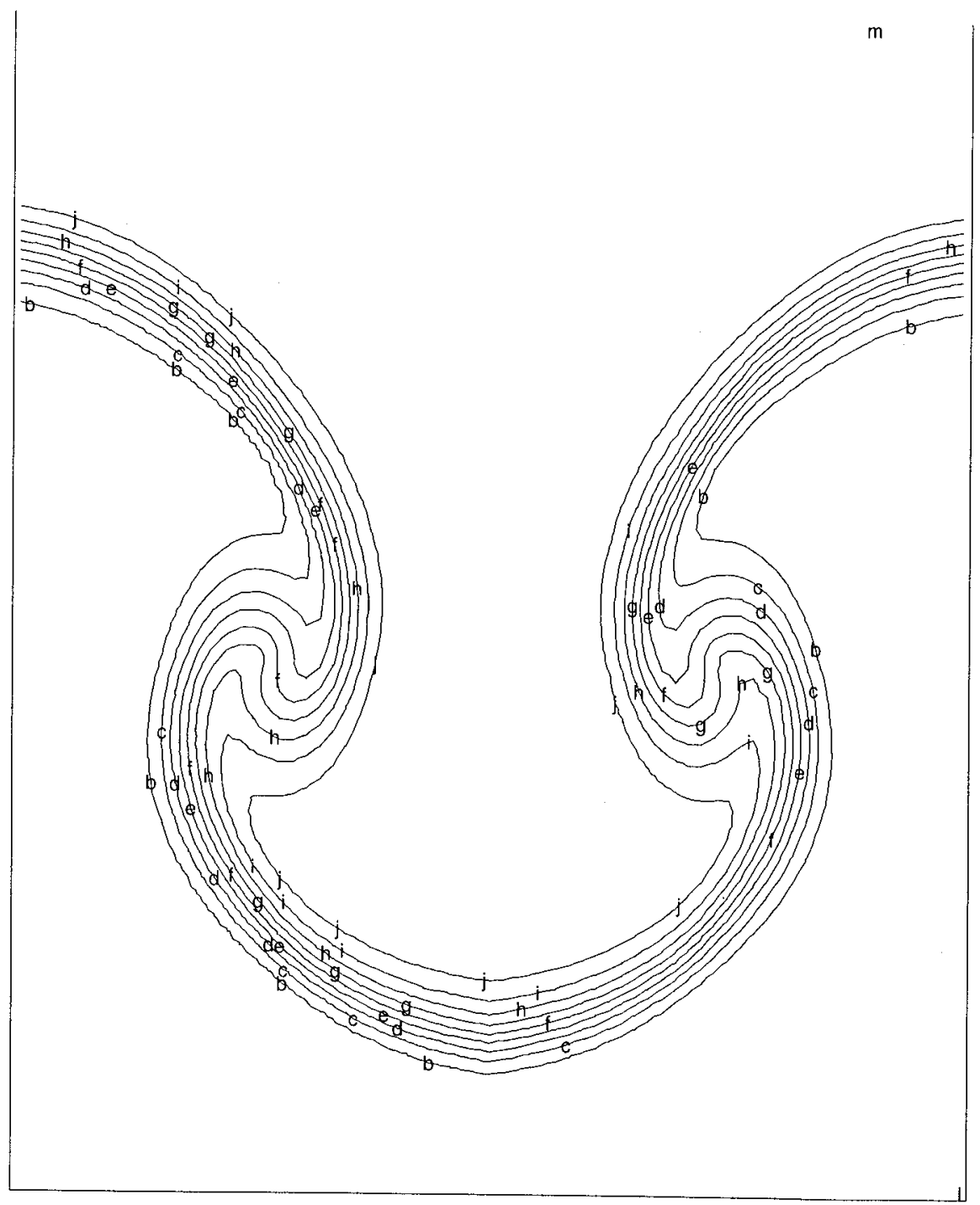

Figure 4: Mass fraction contours for species 1 at $40 \mathrm{~ms}$ using donor cell differencing. 


\section{Density cycle $=6985 \mathrm{t}=4.000385 \mathrm{D}-02 \mathrm{dt}=4.347733 \mathrm{D}-06$ $\max =4.075086 \mathrm{D}-02 \mathrm{~min}=4.105870 \mathrm{D}-03 \mathrm{dq}=3.664499 \mathrm{D}-03$}

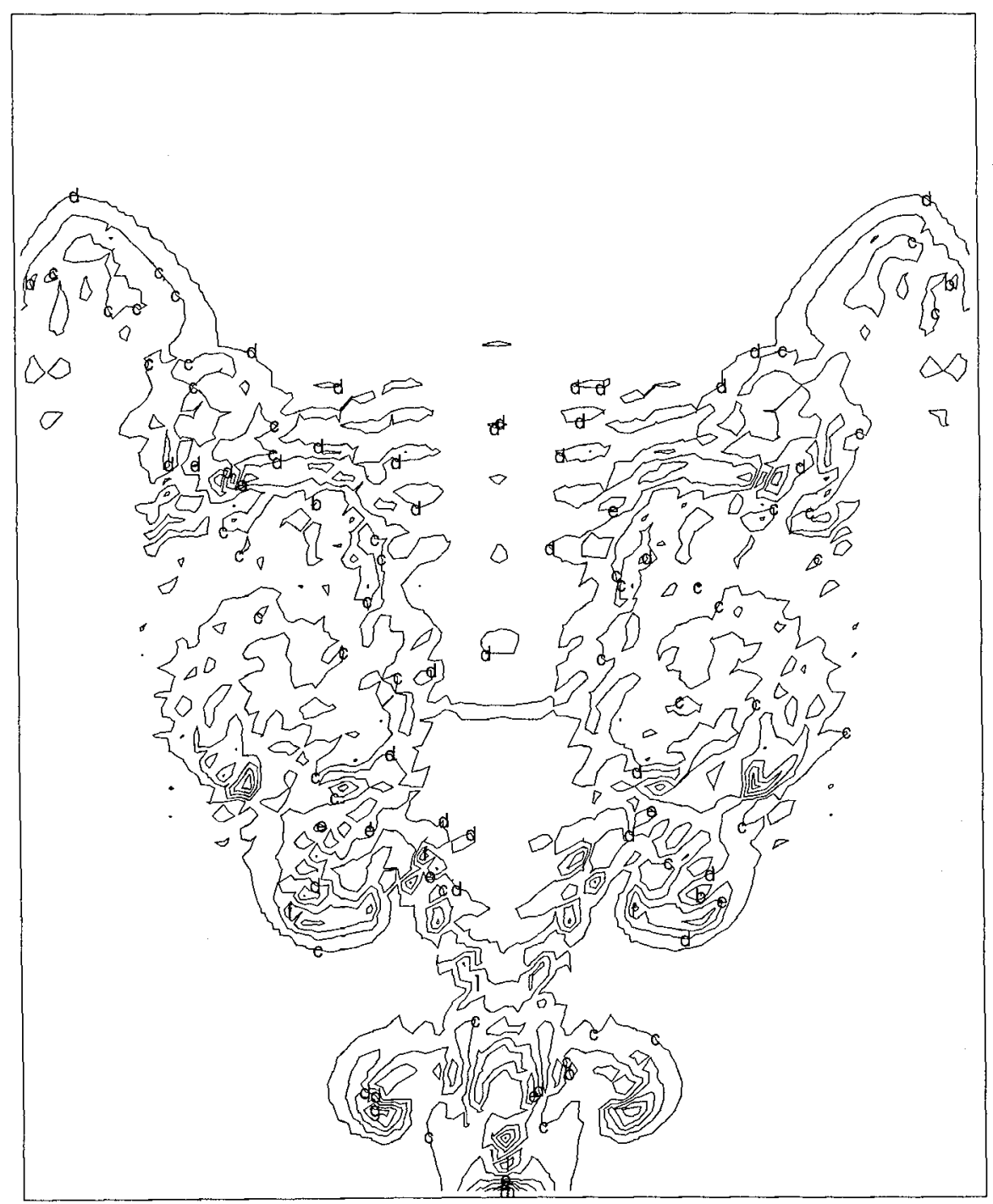

Figure 5: Total density contours at $40 \mathrm{~ms}$ using the tensor viscosity method. 


\section{Temper cycle $=6985 \mathrm{t}=4.000385 \mathrm{D}-02 \mathrm{dt}=4.347733 \mathrm{D}-06$ $\max =1.078109 \mathrm{D}+03 \mathrm{~min}=1.108626 \mathrm{D}+02 \mathrm{dq}=9.672468 \mathrm{D}+01$}

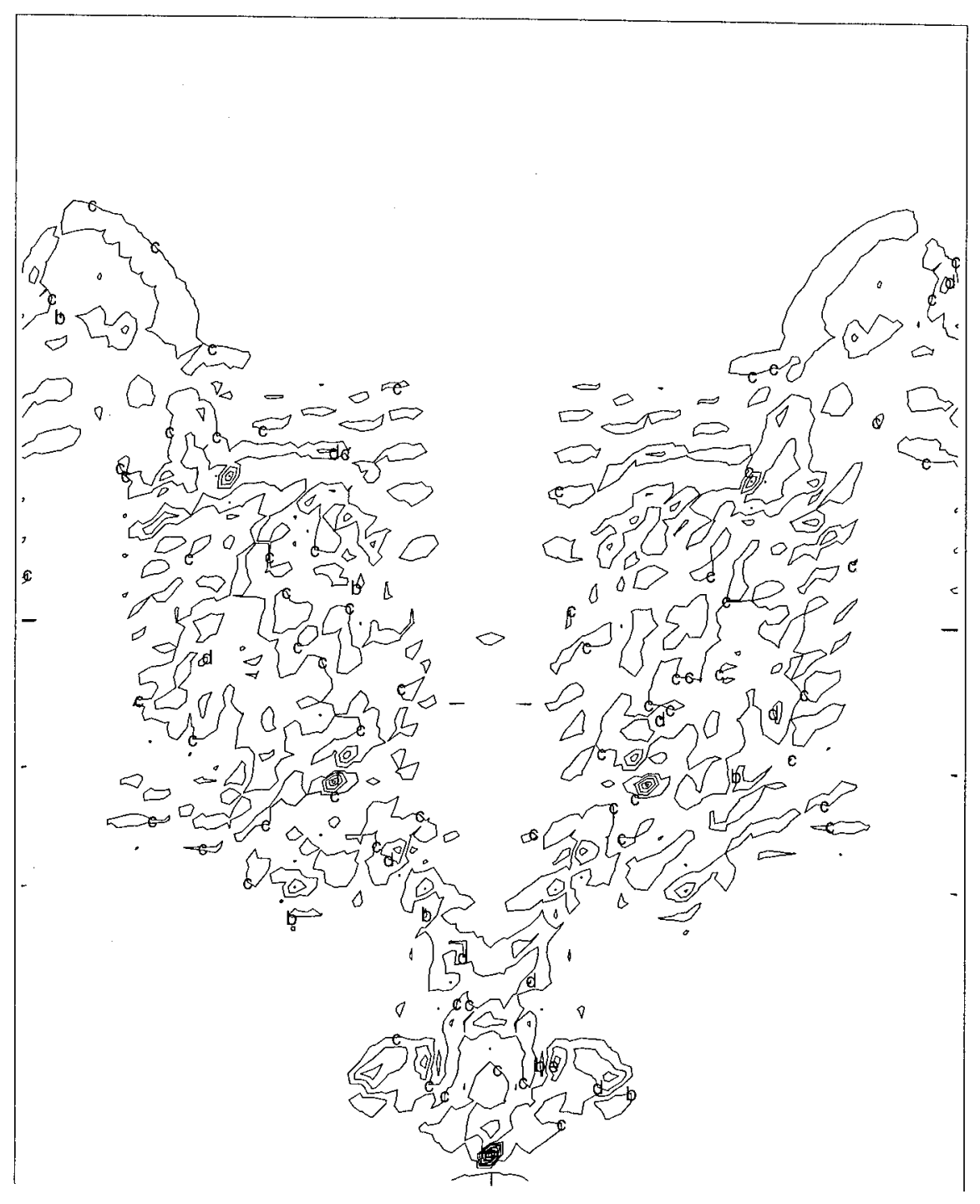

Figure 6: Temperature contours at $40 \mathrm{~ms}$ using the tensor viscosity method. 


\section{Y 1 cycle $=6985 \mathrm{t}=4.000385 \mathrm{D}-02 \mathrm{dt}=4.347733 \mathrm{D}-06$ $\max =1.000111 \mathrm{D}+00 \mathrm{~min}=-5.448470 \mathrm{D}-06 \mathrm{dq}=1.000117 \mathrm{D}-01$}

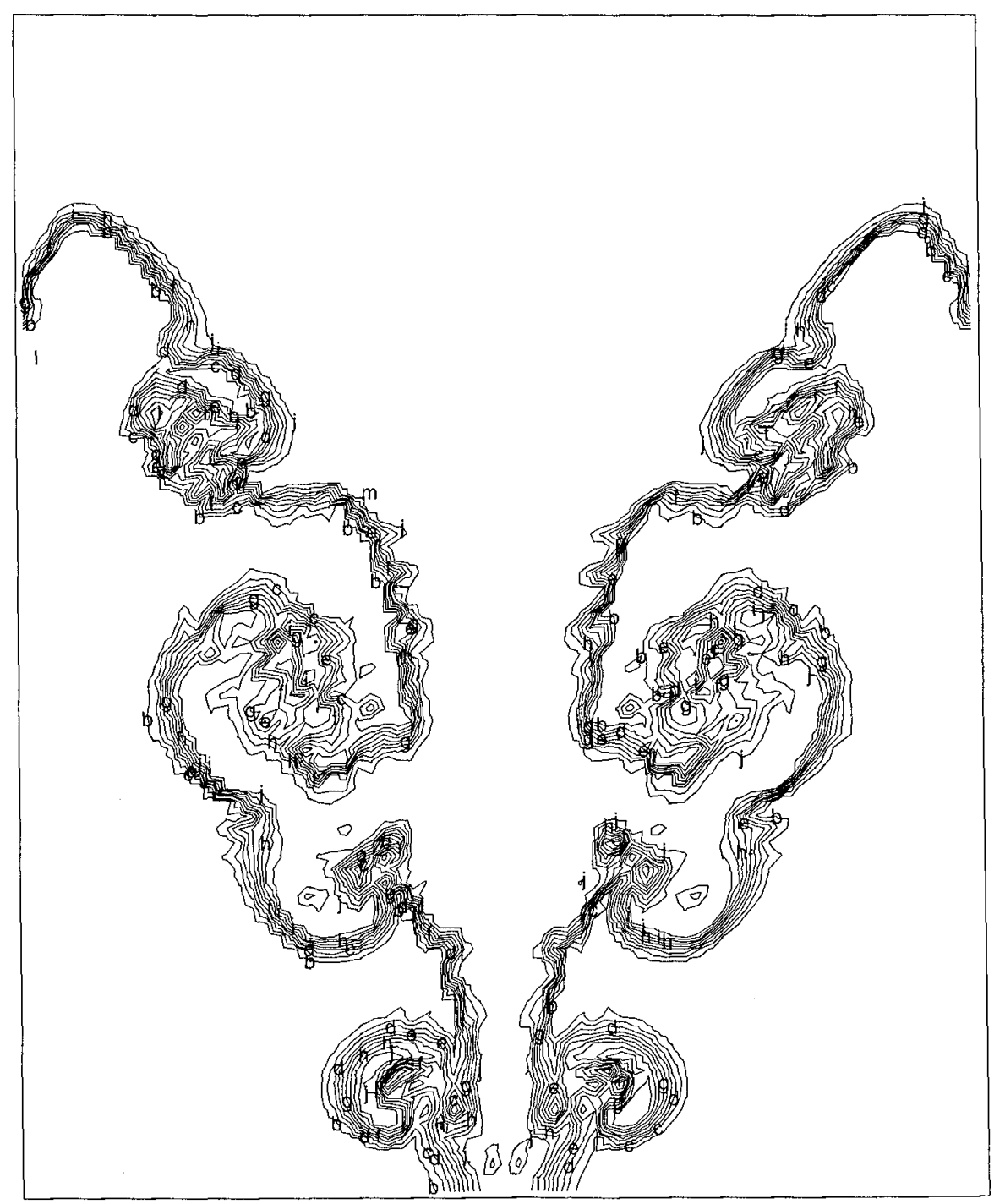

Figure 7: Mass fraction contours for species 1 at $40 \mathrm{~ms}$ using the tensor viscosity mothod. 
Density cycle $=2370 \mathrm{t}=4.001409 \mathrm{D}-02 \mathrm{dt}=1.562439 \mathrm{D}-05$
$\max =1.658974 \mathrm{D}-02 \mathrm{~min}=9.859479 \mathrm{D}-03 \mathrm{dq}=6.730259 \mathrm{D}-04$

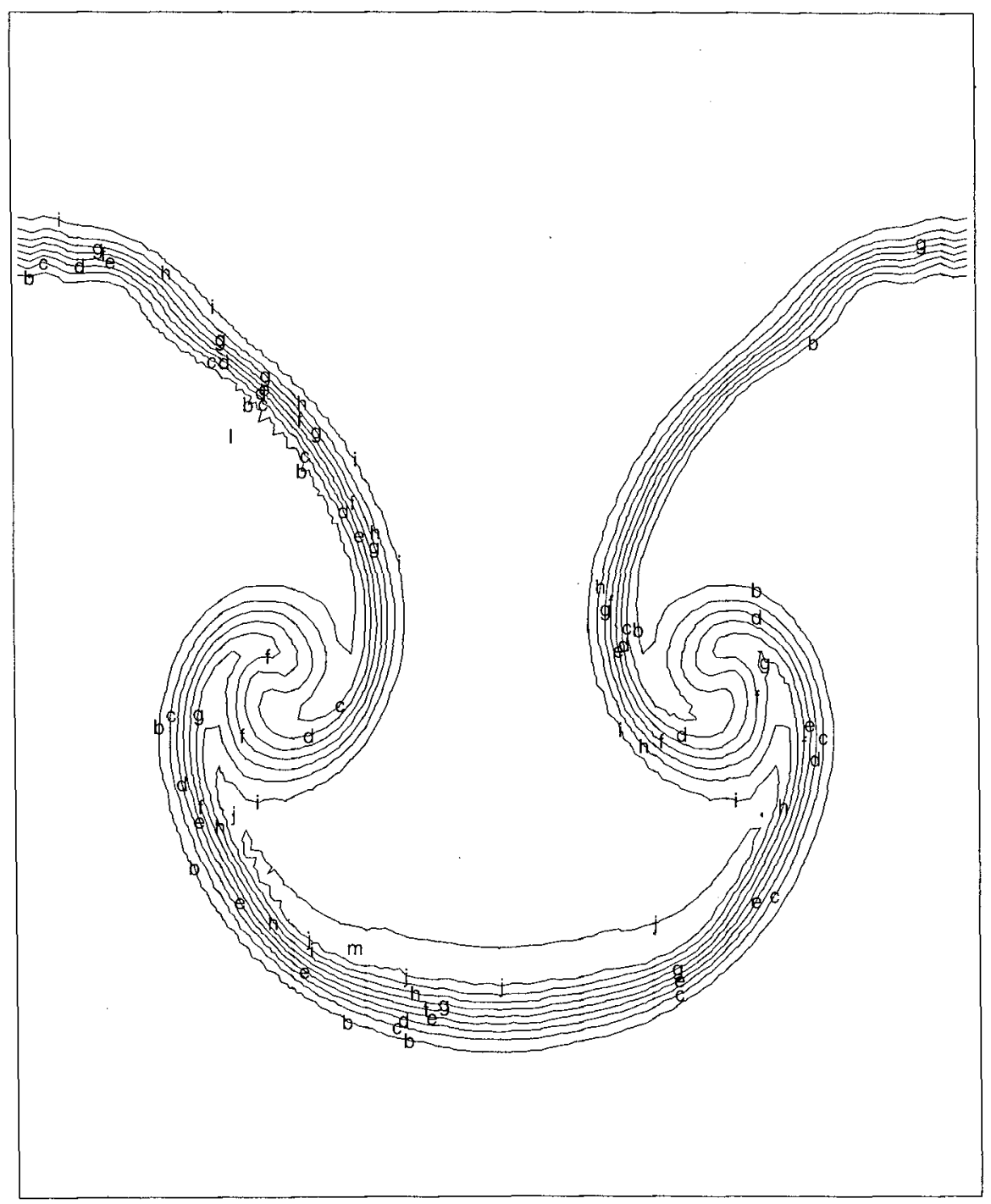

Figure 8: Total density contours at $40 \mathrm{~ms}$ using the tensor viscosity plus CLAM method. 


\section{Temper cycle $=2370 \mathrm{t}=4.001409 \mathrm{D}-02 \mathrm{dt}=1.562439 \mathrm{D}-05$ $\max =2.958701 \mathrm{D}+02 \min =2.722983 \mathrm{D}+02 \mathrm{dq}=2.357177 \mathrm{D}+00$}

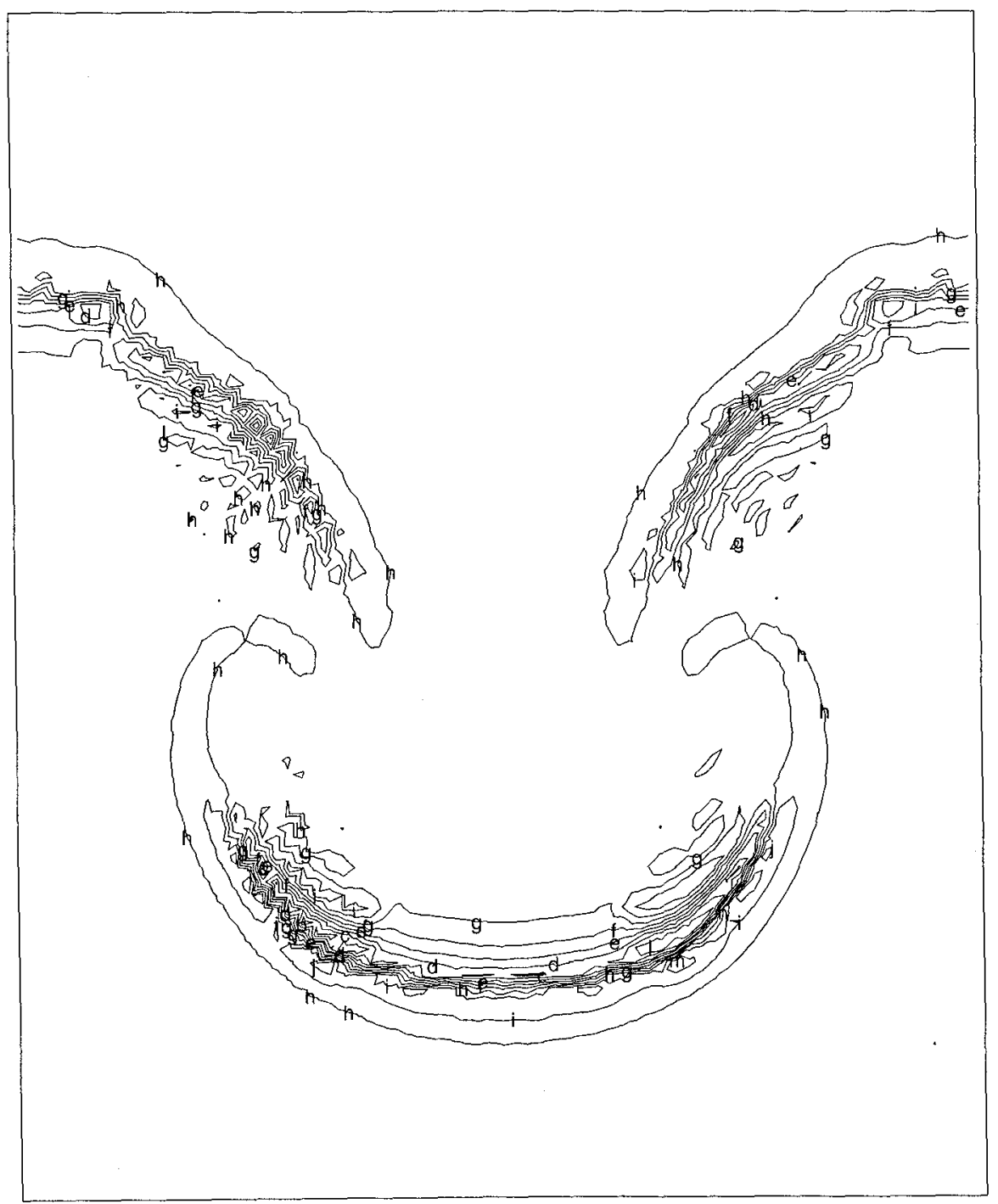

Figure 9: Temperature contours at $40 \mathrm{~ms}$ using the tensor viscosity plus CLAM method. 


\section{Y 1 cycle $=2370 \mathrm{t}=4.001409 \mathrm{D}-02 \mathrm{dt}=1.562439 \mathrm{D}-05$ \\ $\max =1.000004 \mathrm{D}+00 \mathrm{~min}=-4.316624 \mathrm{D}-06 \mathrm{dq}=1.000009 \mathrm{D}-01$}

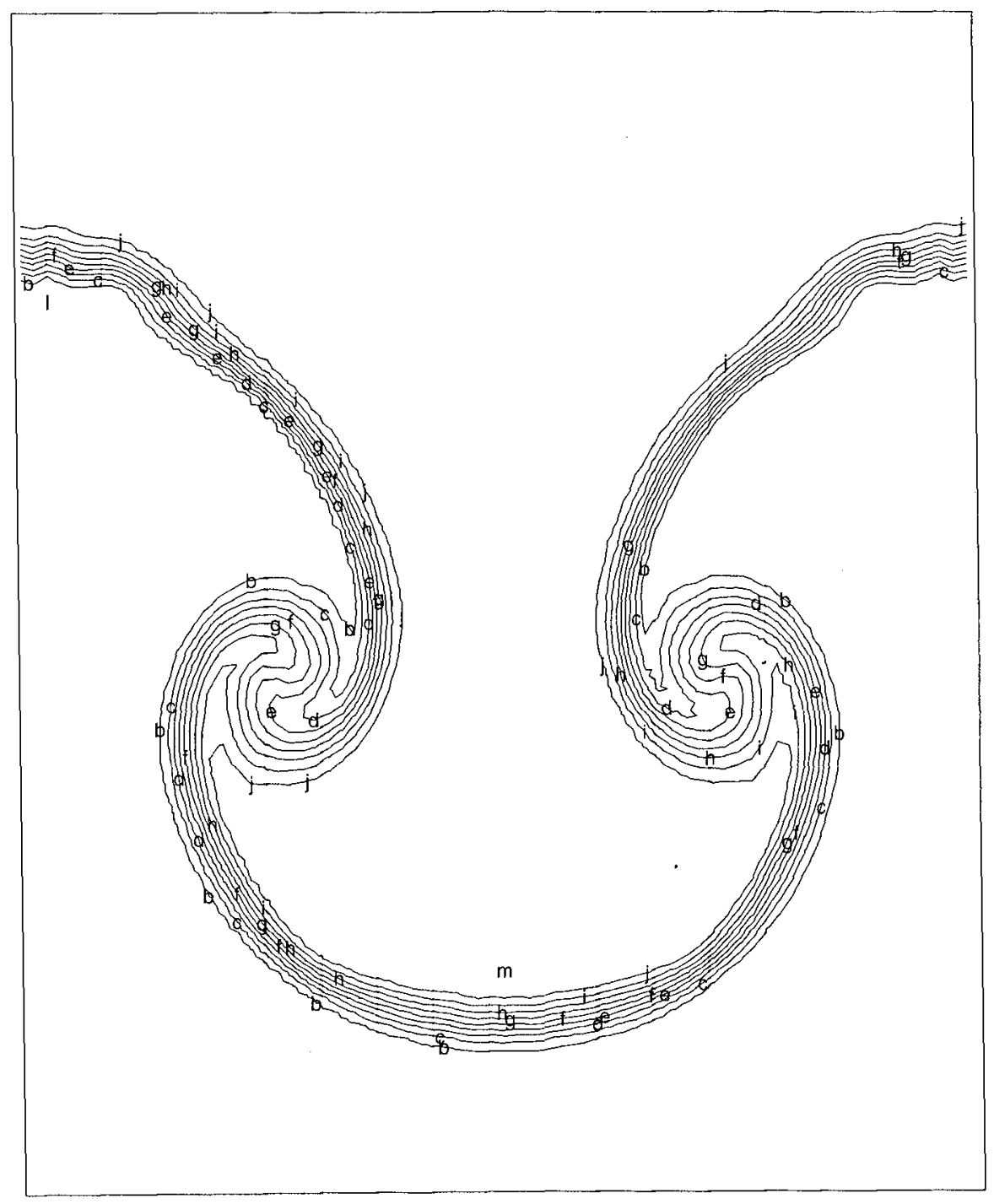

Figure 10: Mass fraction contours for species 1 at $40 \mathrm{~ms}$ using the tensor viscosity plus CLAM method. 


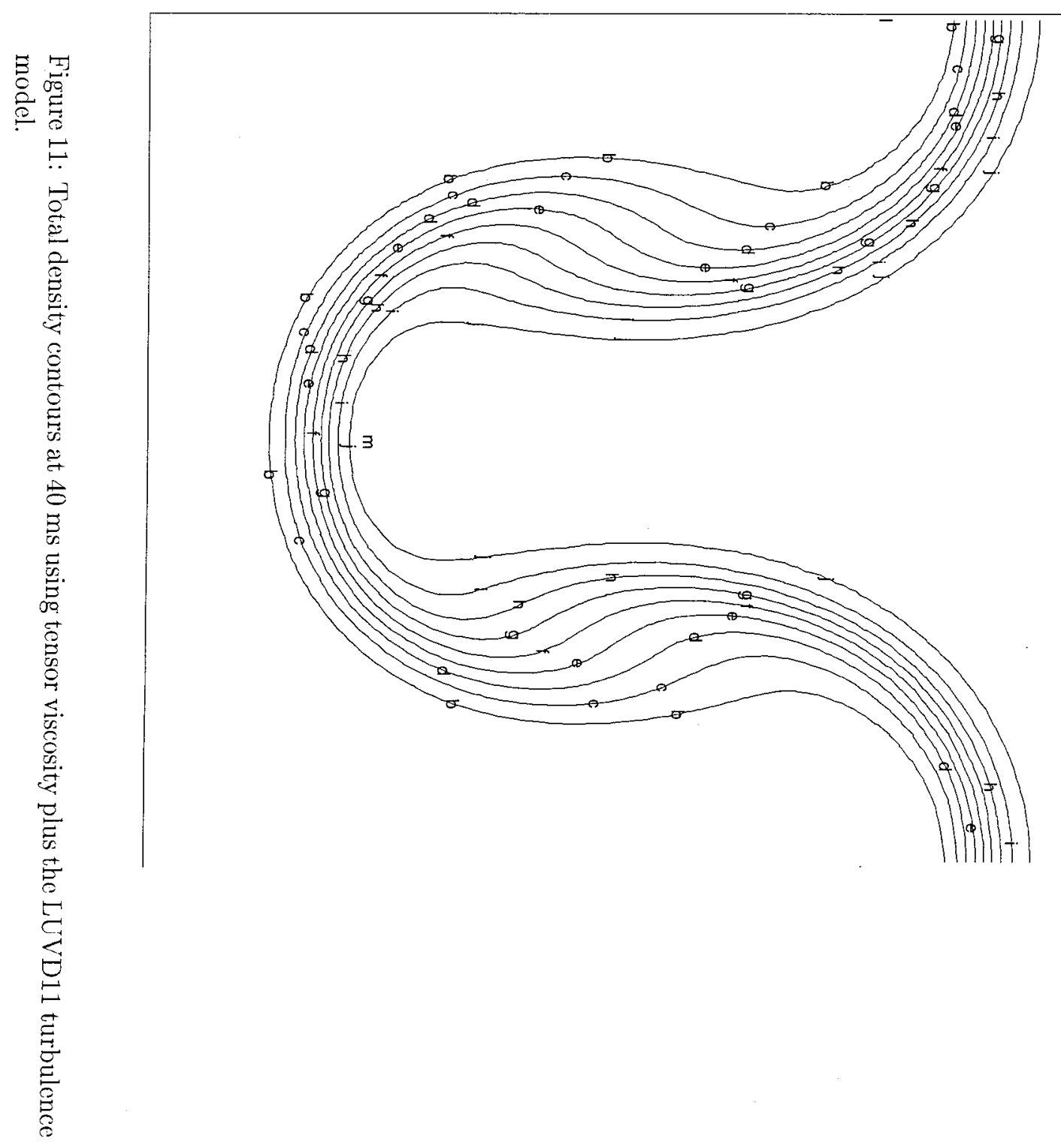

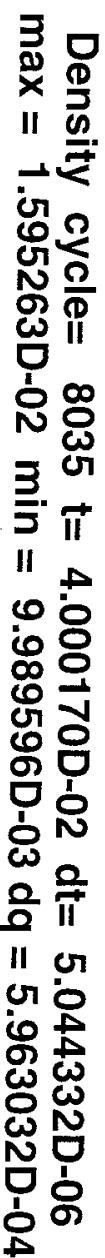




\section{Temper cycle $=8035 \mathrm{t}=4.000170 \mathrm{D}-02 \mathrm{dt}=5.044332 \mathrm{D}-06$ $\max =2.889788 \mathrm{D}+02 \min =2.831951 \mathrm{D}+02 \mathrm{dq}=5.783704 \mathrm{D}-01$}

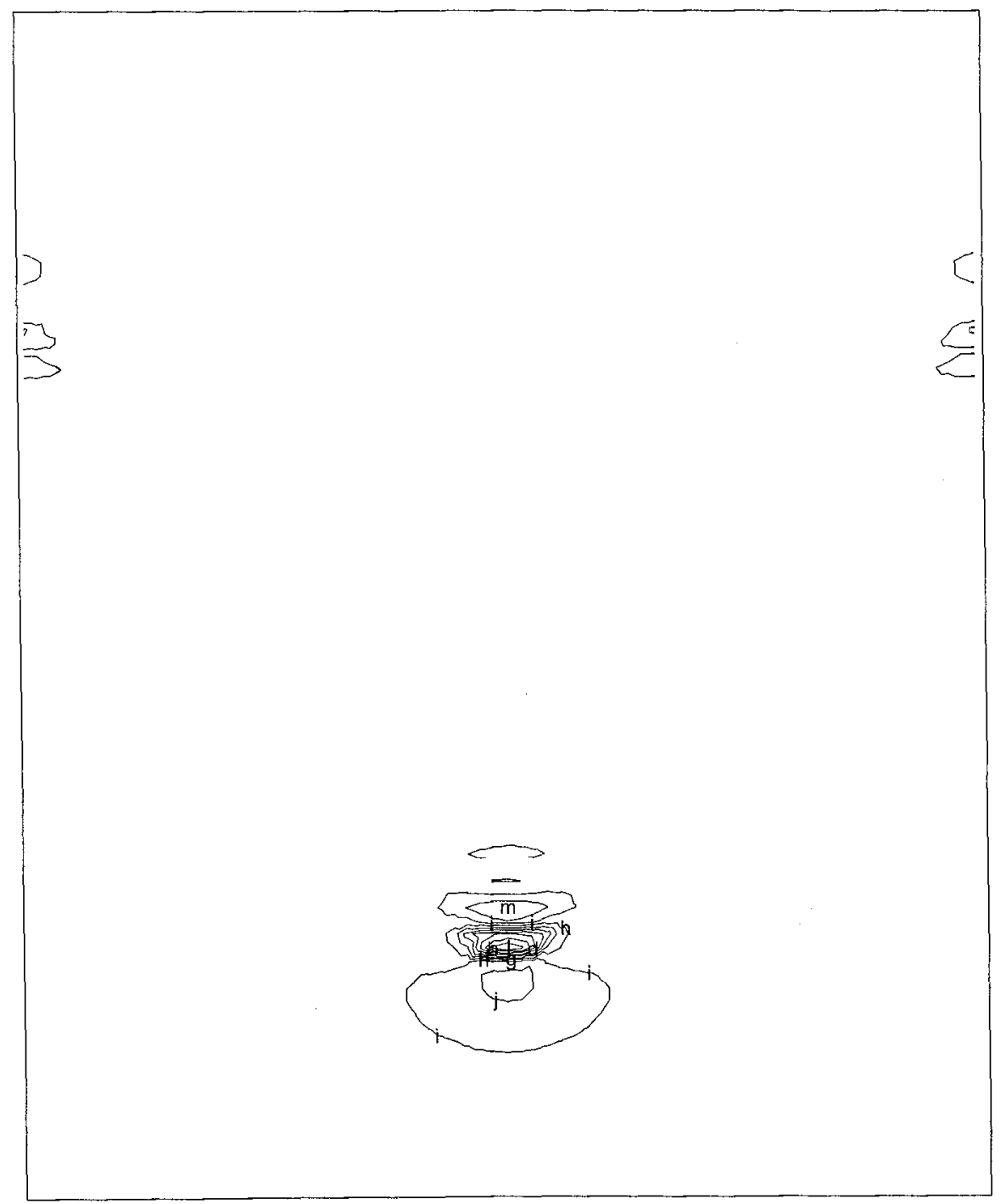

Figure 12: Temperature contours at $40 \mathrm{~ms}$ using tensor viscosity plus the LUVD11 turbulence model. 


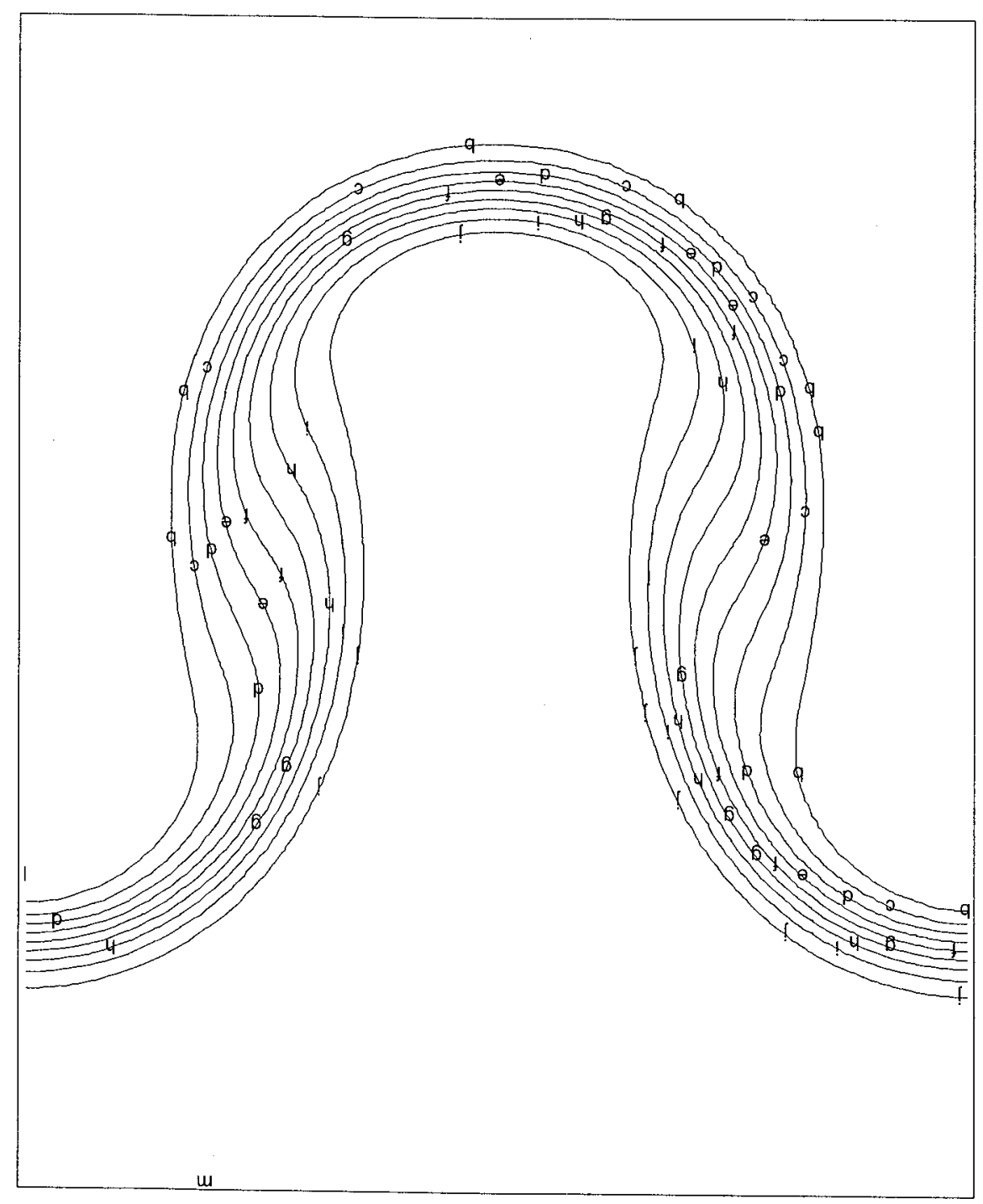

\section{$10-0000000^{\circ} \mathrm{L}=\mathrm{bp} 00+0000000^{\circ} 0=\mathrm{u} u \mathrm{u} 00+0000000^{\circ} \mathrm{L}=\mathrm{xem}$

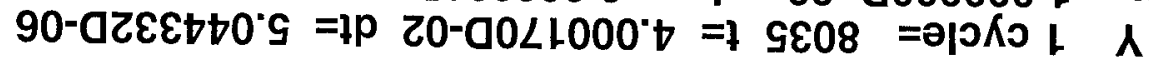




\section{Density cycle $=7175 \mathrm{t}=4.000258 \mathrm{D}-02 \mathrm{dt}=5.331587 \mathrm{D}-06$ $\max =1.621820 \mathrm{D}-02 \min =9.939485 \mathrm{D}-03 \mathrm{dq}=6.278713 \mathrm{D}-04$}

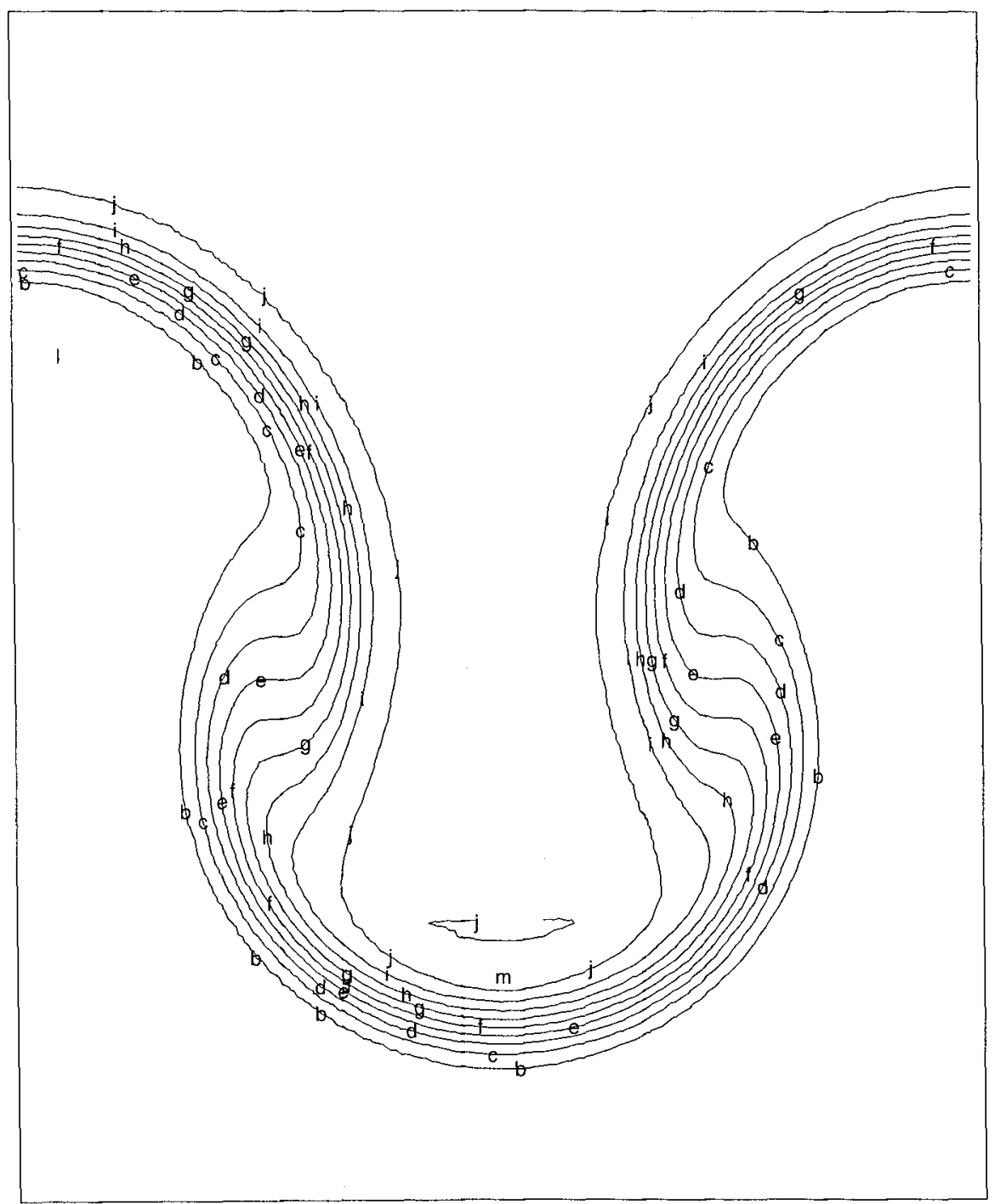

Figure 14: Total density contours at $40 \mathrm{~ms}$ using tensor viscosity plus the LUVD11 model plus CLAM. 


\section{Temper cycle $=7175 \mathrm{t}=4.000258 \mathrm{D}-02 \mathrm{dt}=5.331587 \mathrm{D}-06$ $\max =2.911324 \mathrm{D}+02 \min =2.785546 \mathrm{D}+02 \mathrm{dq}=1.257785 \mathrm{D}+00$}

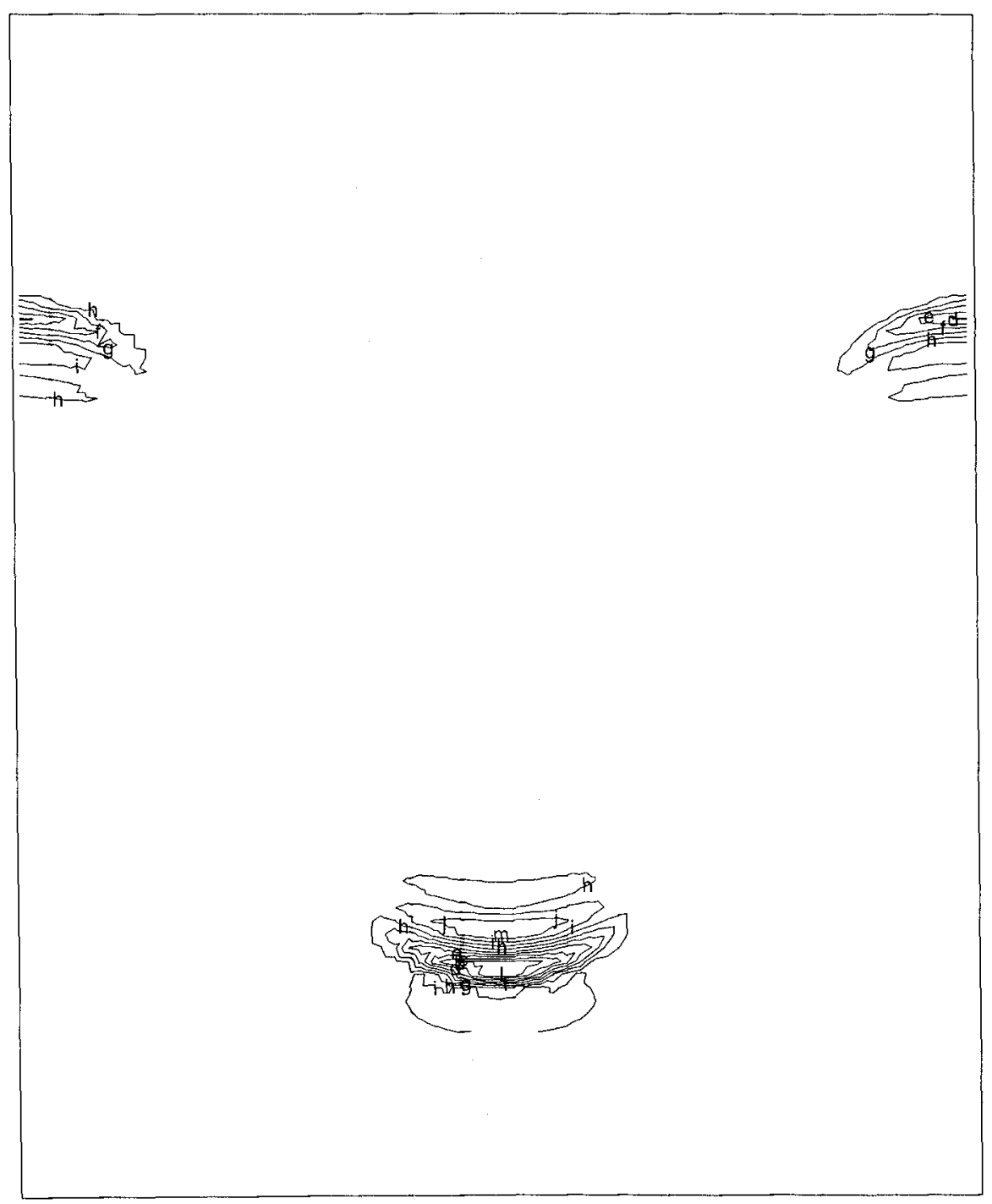

Figure 15: Temperature contours at $40 \mathrm{~ms}$ using tensor viscosity plus the LUVD11 model plus CLAM. 


\section{$\mathrm{Y} 1 \mathrm{cycle}=7175 \mathrm{t}=4.000258 \mathrm{D}-02 \mathrm{dt}=5.331587 \mathrm{D}-06$ $\max =1.000014 \mathrm{D}+00 \mathrm{~min}=-2.155212 \mathrm{D}-05 \mathrm{dq}=1.000035 \mathrm{D}-01$}

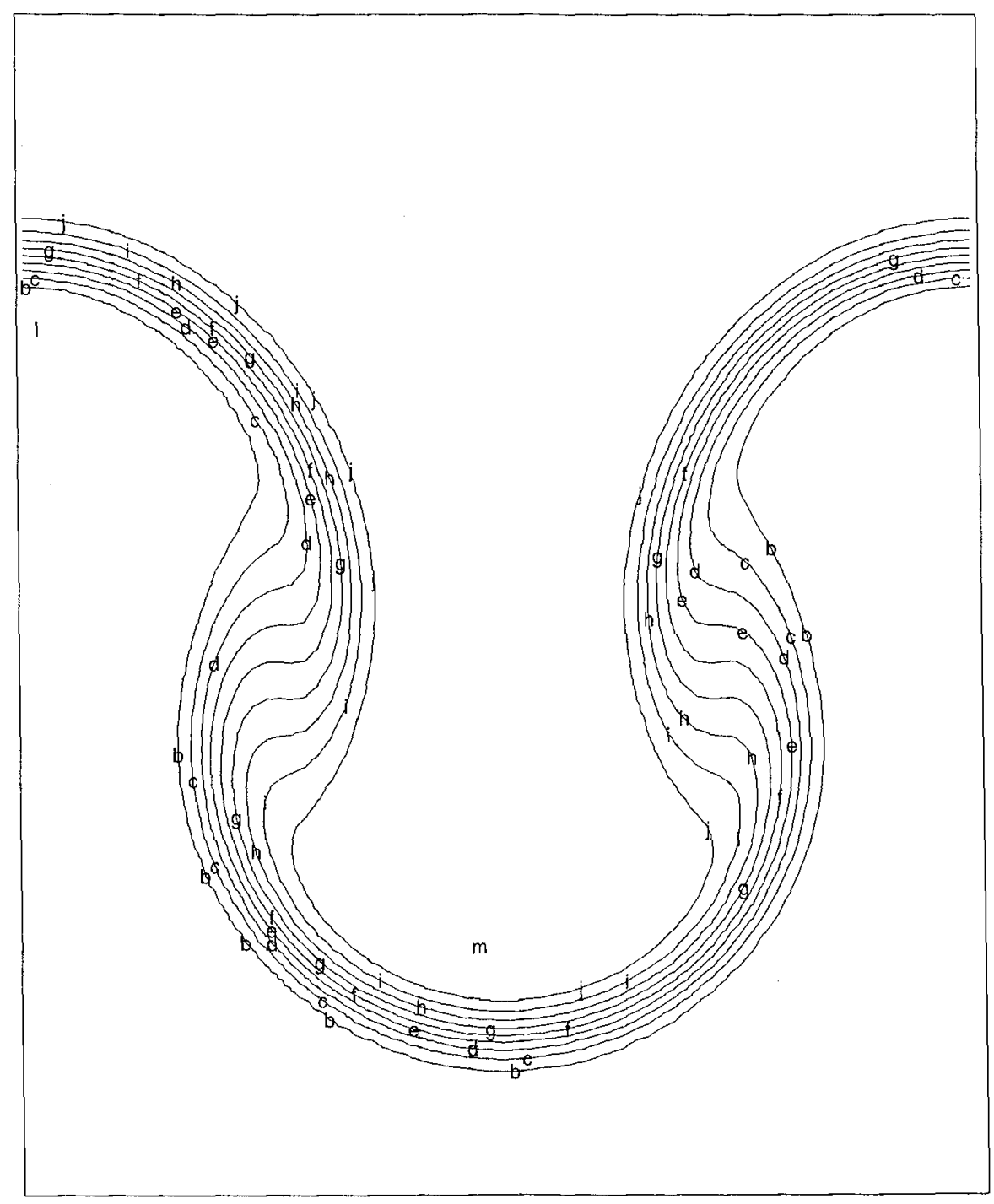

Figure 16: Mass fraction contours for species 1 at $40 \mathrm{~ms}$ using tensor viscosity plus the LUVD11 model plus CLAM. 


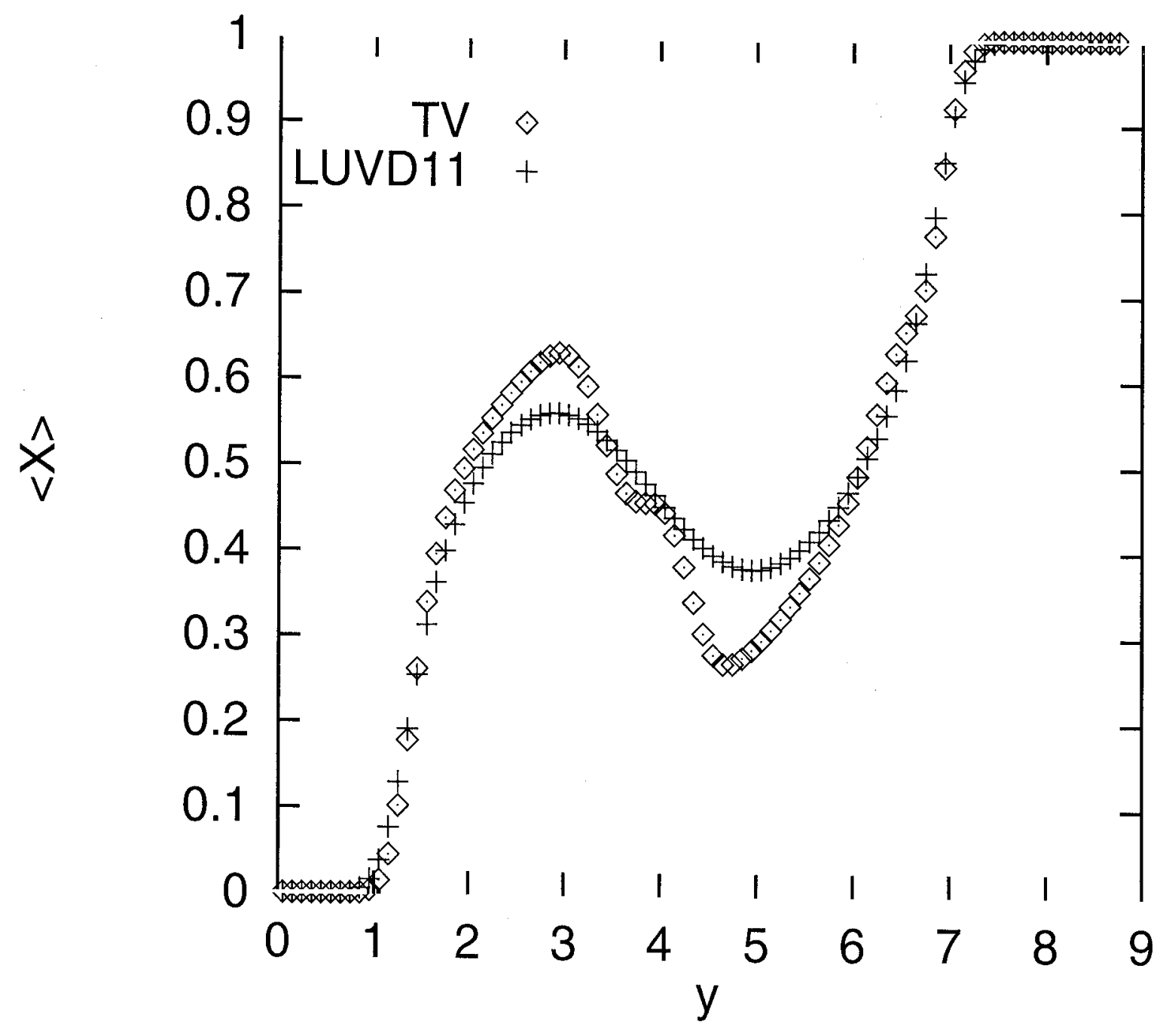

Figure 17: Horizontally averaged mass fraction of species 1 at $40 \mathrm{~ms}$ using the CLAM method, with and without the turbulence model. 